l'Extrême-Orient. La culture du palmier à huile va certainement prendre une plus large place dans la production agricole du Cameroun: la station de Dibamba continuera à répondre à l'appel du gouvernement camerounais, en mettant à la disposition des producteurs le matériel végétal et les techniques modernes assurant les meilleurs rendements. D'ailleurs il est envisagé de promouvoir d'autres cultures oléagineuses comme le cocotier ou l'arachide.

\title{
DIE STAUANLAGEN UND DIE GEOGRAPHIE
}

\author{
EDWIN FELS
}

Stauanlagen errichtet der Mensch seit weit zurückliegenden Zeiten. Aber erst im industriellen Zeitalter, genau besehen erst im 20. Jahrhundert, sind sie an Zahl und Größe so riesig gewachsen, daß sie zum weitaus sinnfälligsten Ausdruck der menschlichen Einwirkung auf die Festlandgewässer geworden sind und wie kaum ein anderes Ergebnis menschlicher Arbeit das Gesicht der Erdoberfläche verändern. Das Endergebnis ist noch längst nicht vorauszusehen, da heute zahllose Werke im Bau sind und geplant werden. Fest steht nur, daß sie eine so wichtige und weitverbreitete Erscheinung geworden sind, daß der Geograph nicht achtlos an ihnen vorübergehen darf, sondern sich ernsthaft mit ihrem Wesen, ihren Eigenschaften und ihren Wirkungen befassen muß.

Der Zeitpunkt einer geographischen Betrachtung der Stauanlagen ist heute insofern günstig, als kürzlich eine umfassende Bestandsaufnahme veröffentlicht wurde, die über rund 10000 Stauanlagen zuverläßig Auskunft gibt. Es handelt sich um das von der International Commission on Large Dams (ICOLD, Sitz Plaris 9e, rue Saint-Georges 51) herausgegebene World Register of Dams (Registre Mondial des Barrages), Paris 1964 (4 Lose-Blatt-Bände, ergänzbar. Bd. 1: Europa einschließlich Sowjetunion und Amerika [ohne USA]. Bd. 2: USA. Bd. 3: Asien, Afrika, Australien. Bd. 4: Sperren im Bau und geplant). Wir finden in diesem Werk nach dem Stichtag vom 31. 12. 1962 Angaben aus 48 Mitgliedländern über alle Anlagen, deren Bauhöhe mindestens 15 Meter (5o feet) beträgt, geordnet nach den Jahren ihrer Fertigstellung. Hier ist eine Fülle wissenswerter Daten vereinigt: Name der Sperre, Jahr der Fertigstellung, Name des Flusses, nächste Stadt oder Ortschaft, politischer Bezirk, Bauart der Sperre in neun möglichen Arten, Höhe über dem tiefsten Fundament oder (und) über dem Flußbett, Kronenlänge, Sperreninhalt, Seevolumen, $Z$ wecke, installierte Kraft, Besitzer, Erbauer.

Titel des Werkes, Herausgeber und mitgeteilte Daten vernaten, $\mathrm{da} ß$ es sich um eine Ingcnieurarbeit handelt. Wir begrüßen sie dankbar und benützen sie gerne, bedauern aber lebhaft, daß sie längst nicht alle Wünsche und Fragen des Geographen berïcksichtigt, die dieser bei Stauanlagen erfüllt und beantwortet wissen möchte. Dem Techniker liegt offenbar nur die Sperre, das Bauwerk am Herzen, während der Stausee, also das eigentlich Geographische, ihm nebensächlich und nur in bezug auf das Seevolumen erwähnenswert erscheint. Für uns aber sind für die Kennzeichnung einer Stauanlage außerdem folgende Begriffe sehr wesentlich: größte Spiegel- und Stauhöhe, Seelänge, Seebreite, Seefläche, Seeumfang, Speicherfaktor. All das finden wir leider in dem sonst so anerkennenswerten World Register nicht. Wir müssen es in mühsamer Kleinarbeit zusammensuchen und bleiben oft genug erfolglos. Dabei darf man aber unterstellen, daß den Ingenieuren alle diese von uns gewünschten Daten wohlbekannt sind, da sie bei der Planung und Ausführung selbstverständlich eine Rolle gespielt haben. So müssen wir Geographen dringlich fordern, daß sie auch veröffentlicht werden und nicht in den Archiven verschwinden. Für uns wäre es wichtig, auch ein World Register of Reservoirs zu besitzen, da ja die Sperren

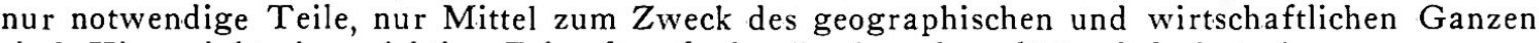
sind. Hier winkt eine wichtige Zukunftsaufgabe. Je eher sie gelöst wird, desto besser.

Mit Bedauern muß beklagt werden, daß es bei dem World Register an dem notwendigen Gefühl für den Zusammenhang der Wissenschaften gefehlt hat. Dieses Bedauern erstreckt sich auch darauf, daß manche Mitgliedstaaten sich in Schweigen gehüllt haben (Bulgarien, Rumänien, Tschechoslowakei, Indonesien, Laos, Marokko), und noch mehr darauf, daß Angaben aus Nichtmitgliedländern fehlen, die in der Stauseewirtschaft eine immerhin beachtliche Rolle spielen, zum Beispiel China, Korea, Iran, Afghanistan, Südafrika, Peru, Sowjetische Besatzungszone Deutschlands und so weiter. Trotzdem ist die Freude über das Erscheinen des wichtigen 
Standardwerkes groß. Sein Vorläufer soll auch nicht unerwähnt bleiben: das Register of Dams in the United States von T. W. Mermel (New York 1958, 429 S., über 300 vorzügliche Abbildungen), das den Stand vom 1.1.1957 aufzeigt und nach den gleichen Grundsätzen aufgebaut ist.

Bei dieser Sachlage ist es notwendig darzulegen, welch vielseitige Interessen den Geographen mit den Stauanlagen als einer weitverbreiteten Erscheinungsform der Erdoberfläche verbinden. Möchten doch auch die Ingenieure davon Kenntnis nehmen und danach handeln!

\section{Begriffe der Stauanlagen}

Da mit den technischen Ausdrücken allein kein Auskommen ist, müssen Begriffe Geltung erlangen, die den geographischen Bedürfnissen gerecht werden. Sperre und Stausee fassen wir als Stauanlage zusammen. Die Sperren (englisch Dam, auch Storage Dam, französisch Barrage [de retenue], italienisch Diga, spanisch Presa) gliedern sich in Fluß-und Talsperren. Jene liegen in Flachlandflüssen oder sehr weiten Tälern, meist an großen Strömen. Diese finden sich im Bergland oder Hochgebirge, in Talengen, Schluchten oder Klammen, überwiegend an kleineren Flüssen und Nebenflüssen, oft im Quellgebiet oder Oberlauf. Bei Flußsperren gebrauchte man gerne den Begriff Staudamm, der aber ungeeignet ist, da er wohl für den aufgeschütteten Erddamm paßt, nicht aber für die gebaute Staumauer. Der englische Dam kennt diese Unterscheidung nicht und wird verallgemeinert gebraucht. Die Stauseen (englisch Reservoir, auch Storage Reservoir oder schlechthin Lake, französisch Retenue oder Réservoir, italienisch Serbatoio, spanisch Pantano oder Embalse, portugiesisch Açude, russisch Vodochranilisce) gliedern sich entsprechend in Fluß- und Talseen wie in der physischen Geographie. Unechte Stauseen sind die häufig gestauten Naturseen.

\section{Kennzeichnung der Sperren}

Bei der Bauart der Sperren genügen den Geographen, im Gegensatz zu den vielen Unterscheidungen der Techniker, zwei Begriffe, die sich im Aussehen scharf voneinander abheben: Mauern und Erddämme. Jene sind steile, oft fast senkrechte Betonmassen von geringer Dicke, diese breite, sanft geböschte, busch- und baumbestandene Gebilde, deren Volumen ein Mehr-, ja Vielfaches der Mauern ist. Die Mauer ist die Bauform des Gebirges, besonders des Hochgebirges, und der Engtäler, der Erddamm die des Flachlandes, aber auch nur dort, wo reichlich Füllmaterial und vor allem auch Ton für die Aufschüttung des undurchläßigen Kerns zur Verfügung stehen. Technischer Fortschritt und geringere Kosten begünstigen neuerdings den Erddammbau, der heute bei etwa 50 Prozent der Neubauten Anwendung findet. Sehr zahlreich sind aber auch gemischte Typen, da zumindest das Kraftwerk stets in einem Mauerbau liegt. Der Geograph begrüßt diesen Wandel, da Mauern immer einen Schlag ins Gesicht der Natur bedeuten, um so mehr, je höher sie sind, mögen sie auch als technische Wunder gelten. Dämme fügen sich viel besser ins Landschaftsbild ein und entwickeln sich mit der Zeit zu einem wenig auffälligen Wesen, mit dem sich auch der Natur- und Landschaftsschutz abfinden kann.

Bei der Sperrenhöhe interessieren sich Techniker und Geograph für verschiedene Werte. Für jenen ist die Bauhöhe wichtig: vom tiefsten, im Flußbett liegenden Fundament bis zu der stets von einer Straße, einer Eisenbahn oder einem Weg besetzten Krone. Dann und wann gibt er auch die Höhe über dem Flußbett an, was unseren Wünschen näherkommt. Für uns aber ist viel aufschlußreicher die fast nie mitgeteilte Stauhöhe als Unterschied zwischen Unterwasser und höchstem Seespiegel, die nur zufällig und auf Umwegen zu erfahren ist. Die maximale Höhe des Seespiegels über NN ist ebenfalls ein notwendiges Desideratum. Diese Angaben klaffen stets weit auseinander. Immer ist die Stauhöhe geringer als die Bauhöhe, oft sogar sehr erheblich. Bei Mauern ist die Differenz besonders hoch. Aber auch bei Erddämmen treten bedeutende 
Unterschiede auf, wenn etwa durchläßiger Schotter bis auf den festen Fels ausgeräumt werden muß. Da alles von den geologischen Verhältnissen abhängt, lassen sich Gesetzmäßigkeiten nicht ableiten. Die höchsten Sperren sind im allgemeinen Mauern, aber neuerdings treten Erddämme mit ihnen in Wettbewerb.

Höchste Sperren mit mehr als $200 m$ (nach World Register)

Inguri, SU, Transkaukasien

Nurek, SU, Tadschikistan

Grande Dixence, Schweiz, Wallis

Vajont, Italien, Venetien

Mauvoisin, Schweiz, Wallis

Contra, Schweiz, Tessin

Oroville, USA Kalifornien am Feather

Glen Canyon, USA, Arizona/Utah am Colorado

Luzzone, Schweiz, Tessin

Hoover, USA, Nevada/Arizona am Colorado

Bhakra, Indien, Panjab am Sutlej

Manicouagan 5, Kanada, Quebec (St.-Lawrence-

Mündung)

\begin{tabular}{|c|c|c|}
\hline & $\begin{array}{l}\text { Höhe über } \\
\text { tiefstem } \\
\text { Fundament }\end{array}$ & $\begin{array}{l}\text { Höhe über } \\
\text { Flußbett }\end{array}$ \\
\hline Mauer im Bau & 301 & \\
\hline Erddamm im Bau & 300 & \\
\hline Mauer 1962 & 284 & \\
\hline Mauer 1961 & 262 & 259 \\
\hline Mauer 1958 & 237 & \\
\hline Mauer 1965 & 230 & \\
\hline $\begin{array}{l}\text { Erddamm im Bau } \\
\text { (fertig 1968) }\end{array}$ & & 224 \\
\hline Mauer 1964 & & 216 \\
\hline Mauer 1963 & 208 & \\
\hline Mauer 1936 & 221 & 176 \\
\hline Mauer 1965 & 226 & 171 \\
\hline $\begin{array}{r}\text { Mauer im Bau } \\
\text { (fertig 1967) }\end{array}$ & 226 & 160 \\
\hline
\end{tabular}

Die stets angegebene Kronenlänge kennzeichnet die geomorphologische Gestaltung der Landschaft. Sie ist bei Mauern meist gering und kommt oft der Stauhöhe gleich oder bleibt sogar unter ihr. Erddämme dagegen sind in der Regel sehr lang und breit. Den Rekord dürfte der im Bau befindliche Kiew-Erddamm am Dnjepr mit $41185 \mathrm{~m}$ Kronenlänge (davon $285 \mathrm{~m}$ Mauer) halten.

Das Sperrenvolumen ist für den Ingenieur ein überaus wichtiger Begriff, erweckt aber kaum das Interesse des Geographen. Ihm genügt die bereits erwähnte Tatsache, daß alle Erddämme ungleich inhaltsreicher als Mauern sind und daß die Riesenerddämme die Seevolumina großer deutscher Stauseen übertreffen.

\section{Kennzeichnung der Stauseen}

Das Hauptinteresse des Geographen gilt der leider meist nur auf Umwegen zu erfahrenden Seefläche, die am allermeisten das Landschaftsbild bestimmt und auf seine geomorphologische Gestaltung Schlüsse zu ziehen erlaubt. Hierin schlug in den letzten Jahren ein Rekord in rascher Folge den andern.

\section{Größte Stausecflächen von mehr als $1000 \mathrm{~km} 2$}

(nach verschiedenen, für die SU widerspruchsvollen Quellen) zum Vergleich: Bodensee $538,5 \mathrm{~km}^{2}$

\begin{tabular}{lrlr} 
Akosombo & 1965 & Ghana, Volta & 8500 \\
Kuibyschew (W. I. Lenin) & 1955 & SU, Wolga & 6500 \\
Buchtarma & 1960 & SU, Irtysch & 5500 \\
\multicolumn{1}{c}{ In den Stausee wurde der } & Sajsansee (2380 km2) einbezogen & \\
Bratsk & 1961 & SU, Angara & 5500 \\
Elizabeth Lake (Kariba) & 1959 & Rhodesien, Sambesi & 5200 \\
Rybinsk & 1941 & SU, Wolga & 4580 \\
Wolgograd (22. Kongreß) & 1958 & SU, Wolga & 3160 \\
Zimljansk & 1952 & SU, Don & 2700 \\
Krementschug & 1961 & SU, Dnjepr & 2250 \\
Kachowka & 1955 & SU, Dnjepr & 2150 \\
Kama & 1954 & SU, Kama & 1840 \\
New Hogan & 1964 & USA, Kalifornien & 1785 \\
Gorki & 1955 & SU, Wolga & 1750 \\
Garrison & 1960 & USA, Missouri & 1578
\end{tabular}




$\begin{array}{lrlr}\text { Oahe } & \text { um } 1963 & \text { USA, Missouri } & 1522 \\ \text { Gouin (La Loutre) } & 1917 & \text { Kanada, St. Maurice } & 1295 \\ \text { Rincón del Bonete } & 1946 & \text { Uruguay, Rio Negro } & 1140 \\ \text { Nowosibirsk } & 1957 & \text { SU, Ob } & 107 \text {. } \\ \text { Kentucky } & 1944 & \text { USA, Tennessee } & 1056\end{array}$

Diese Liste ist mangels geeigneter Unterlagen unvollständig. Sicher werden Sadd el Ali (Ägypten), Manicouagan 5 (Kanada) und Krasnojarsk (SU) zu den Riesenstauseen gehören (alle im Bau). Wahrscheinlich sind einzureihen Furnas (1962, Brasilien, Minas Gerais), Saratow (im Bau, SU, Wolga), Nurek (im Bau, SU, Tadschikistan), Wotkinsk (1961, SU, Kama). Als unechte Riesenstauseen könnte man solche bezeichnen, die durch geringen Stau natürlicher Seen mächtige Flächen gewinnen.

Dazu gehören :

$\begin{array}{lcc}\text { Irkutsk } & 1956 & 31640 \mathrm{~km}^{2} \text { Baikalsee ca. } 1 \mathrm{~m} \text { gestaut } \\ \text { Oberer Swir } & 1952 & 9700 \mathrm{~km}^{2} \text { Onegasee } \\ \text { Wolchow } & 1926 & 1100 \mathrm{~km}^{2} \text { Ilmensee }\end{array}$

Daß durch die Owen-Falls-Sperre am Victoria Nil (1954) der Victoriasee (66 250 $\mathrm{km}^{2}$ ), wie behauptet wird, um 1 bis $3 \mathrm{~m}$ gestaut wird, ist nicht sicher erwiesen.

Allein die in der Liste aufgezählten 19 Seen, von denen 11 zur Sowjetunion gehören, ergeben eine Flächensumme von ca. $59000 \mathrm{~km}^{2}$. Hier darf eingefügt werden, daß die Gesamtfläche aller USA-Stauseen auf mindestens $60000 \mathrm{~km}^{2}$ geschätzt werden muß. Wenn man für alle Stauseen der Erde 500000 km² rechnet, greift man nicht zu hoch.

Außer der Fläche ist der Geograph dankbar für die Kenntnis von Länge, Breite und Uferlänge der Stauseen, alles Werte, die er sich zusammensuchen muß. Aus Fläche und Uferlänge läßt sich die Umfangsentwicklung berechnen, die angibt, um wieviel die Uferlänge größer ist als der Umfang des flächengleichen Kreises. Sie liefert die beste Aussage über das Ausmaß der Gliederung.

Für den Techniker und den Geographen gleichermaßen belangreich ist der Stauraum oder das Seevolumen. Auf ihm beruhen die Leistungsfähigkeit und die wirtschaftliche Bedeutung der Stauseen. Geographisch am wichtigsten ist der Gesamtstauraum, während für die Wirtschaftlichkeit der Nutzstauraum maßgebend ist, der stets, ja oft sehr erheblich kleiner ist als jener. Oft wird auch ein inaktiver oder toter Stauraum angegeben, der für Schlammablagerung bestimmt ist und hohe Werte einnehmen kann. Ist doch die Sinkstofführung der Flüsse für alle Stauseen - regional freilich in sehr verschiedenem $\mathrm{Maße}$ - ein bedrohliches Problem. Sie kann unter Umständen schon nach 100 Jahren zur Zuschüttung eines Sees führen. Neuere Untersuchungen ergaben jedoch, daß all zu großer Pessimismus nicht am Platz ist. Die Tennessee Valley Authority (TVA) ließ von Anfang an die Sedimentation in ihren Stauseen messend beobachten. So wurde im Norris-See (erbaut 1936, am Clinch, 3166 Mio. $\mathrm{m}^{3}, 163 \mathrm{~km}^{2}$ ) festgestellt, daß sich in 24,4 Jahren bis August 1960 nur 20,7 Mio. $\mathrm{m}^{3}$ oder 0,6\% des Stauraumes angesammelt haben, ein Wert, der sich um 3,7 Mio. $\mathrm{m}^{3}$ unter dem Betrag hielt, mit dem man anfangs gerechnet hatte. Das ist doch sehr ermutigend. Man bedenke: Je mehr in einem Flußgebiet die Stauseen an Zahl und Größe zunehmen, je gründlicher man ihre Zuflüsse verbaut und kleine Seen vorschaltet, je eifriger man Kahlschläge aufforstet, um so mehr schwächt man die Erasion und senkt die Sinkstofführung. Die TVA ist das Beispiel einer voll geglückten Bemühung, das dazu ermutigt, in anderen Flußgebieten (Missouri, Columbia, Texas) ebenso vorzugehen. So kümmert man sich weder in den USA noch sonst in der Welt um solche pessimistischen Vorbehalte, sondern baut unermüdlich neue Riesensperren.

Beim Stauraum muß man an den Begriff des Speicherfaktors erinnern, der das Verhältnis des mittleren Jahreszuflusses zum Gesamtstauraum bezeichnet. Faktor 3 bedeutet also, daß der Zufluß von drei Jahren den See füllt. 
Zum Vergleich: Bodensee $48+32$ Mio. $\mathrm{m}^{3}$

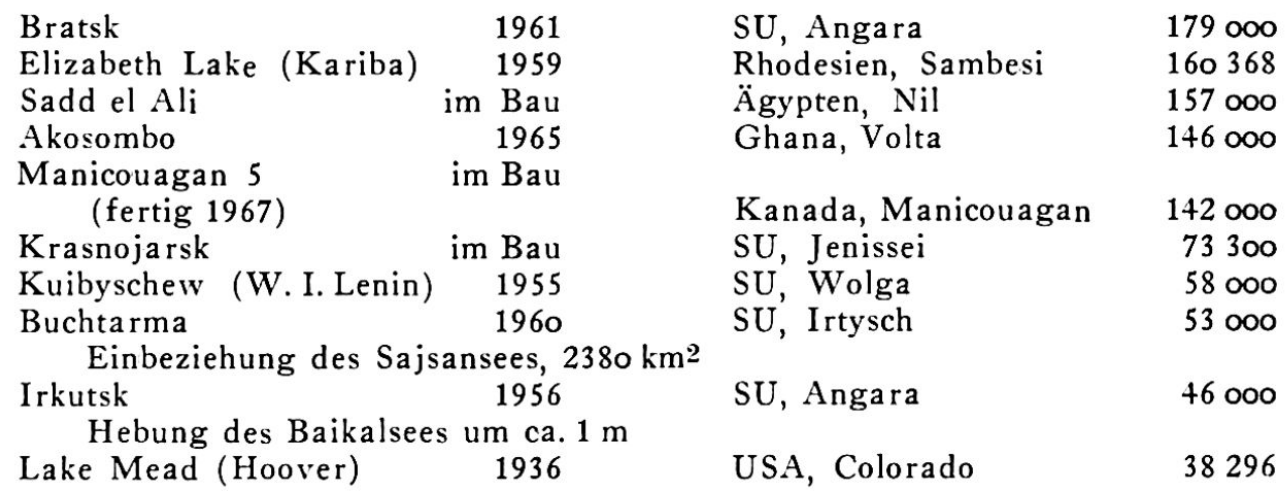

Diese 10 Riesen, bei denen der starke Anteil der Sowjetunion auffällt, stauen zusammen $1053 \mathrm{~km}^{3}$. Für alle Stauseen der Erde dürfte die Schätzung von $5000 \mathrm{~km}^{3}$ nicht allzu weit von der Wirklichkeit abliegen. Wenigstens für die im World Register geführten etwa 10000 Seen die Volumensumme festzustellen, wäre eine dankenswerte Arbeit.

Diese an Fläche und Volumen mächtigen künstlichen Wasseransammlungen verdienen die höchste Beachtung der Geographen, denn sie üben die gleichen Wirkungen aus wie Naturseen. Ihr Einfluß auf das örtliche Klima ist bedeutend und wird von vielen meteorologischen Stationen erforscht. Er erstreckt sich nicht nur auf die nächste Umgebung, sondern - zumal durch weitgedehnte Bewässerungsanlagen - über sehr große weitere Flächen. Sehr interessant ist in stauseereichen Ländern der Vergleich mit den Niederschlägen. Die Stauseen der USA zum Beispiel, können heute etwa ein Achtel des gesamten USA-Niederschlags in ihren Becken aufnehmen. Der Einfluß auf die Wasserführung der Flüsse bedarf dringend einer genauen Untersuchung. Nicht zu übersehen sind die biologischen Auswirkungen, findet doch die Fisch- und Vogelwelt ideale Bedingungen. So wurden am Lake Mead am Colorado mehr als 230 Vogelarten heimisch. Von den anthropogeographischen Auswirkungen wird noch die Rede sein.

\section{Die Gestalt der Stauseen}

Die wichtigste Eigenschaft ist die erstaunlich hohe Gliederung, deren Ursache in der (Plötzlichkeit) der Entstehung der Stauseen liegt. Die Uferlinie zeigt eine ursprüngliche und völlig unveränderte Frische. So sahen die postglazialen Seen unmittelbar nach dem Rückzug der Gletscher aus. Seitdem aber machte die Zuschüttung von allen Seiten solche Fortschritte, daß die Uferlinie weitgehend ausgeglichen wurde und heute einen stark verminderten Gliederungswert aufweist. Die Stauseen aber schmiegen sich jeder kleinsten Geländefalte an und dringen tief in jedes Seitental ein. So zeigen sie riesige Umfangsentwicklungen, denen gegenüber selbst Maximalwerte von Naturseen weit zurückfallen. Hier folgen einige Spitzenbeispiele aus den USA:

$\begin{array}{lccc}\text { Stausee und Staat } & \begin{array}{c}\text { Fläche } \\ \mathrm{km}^{2}\end{array} & \begin{array}{c}\text { Uferlänge } \\ \mathrm{km}\end{array} & \begin{array}{c}\text { Umfangs- } \\ \text { entwicklung }\end{array} \\ \text { Lake of the Ozarks, Missouri } & 242 & 2100 & 38,1 \\ \text { Kentucky, Kentucky/Tennessee } & 1056 & 3830 & 33,2 \\ \text { Chickamauga, Tennessee } & 159 & 1304 & 29,2 \\ \text { Wheeler, Alabama } & 276 & 1711 & 29, \mathrm{o}\end{array}$

Zum Vergleich: Lago di Lugano 3,65, Bodensee 3,46, Vierwaldstättersee 2,91

Maßgebend ist die geomorphologische Formung des Geländes, in das die Stauseen eingebettet sind. Höchste Werte scheinen in Rumpfgebirgen, Mittelgebirgen und welli- 
gen Tafelländern aufzutreten. Flußseen zeigen einfachere Umrißformen als Talseen. Sie bilden sehr lange, stark verbreiterte Flußschläuche mit geringen seitlichen Ausbuchtungen, besonders deutlich an Fremdlingsflüssen, die ein Trockengebiet durchziehen (Nil, Indus). Talseen können auch Hunderte von Kilometern lang und erheblich breit sein ; aber sie sind mannigfaltig gegliedert und dringen in weitverzweigten /Ingressionsbuchten> tief in die Seitentäler ein.

So sind gealterte Naturseen und jugendliche Stauseen durchaus verschiedenartige Gebilde, die auch abgesehen von Gestalt und Gliederung in vielen Beziehungen andersartig sind. Die Maximaltiefe liegt natürlich an der Sperre und nimmt mehr oder weniger gleichmäßig zum oberen Ende hin ab. Unvermeidlich sind oft sehr kräftige Spiegelschwankungen, die unter Umständen mehrere Zehner von Metern betragen können, aber keineswegs müssen. Diese Schwankungen beeinflussen die Wellenarbeit am Ufer und verursachen eine auffällige Kleinterrassierung. Völlig anders vollzieht sich der Abfluß, der auf verschiedenen Wegen und in jeder Höhe angeordnet werden kann. Selbstverständlich sind deshalb auch die thermischen und chemischen Eigenschaften andersartig. Leider treten hin und wieder auch Katastrophen auf, die durch das Bersten von Sperren zur plötzlichen Entleerung des Beckens führen und Menschenverluste und Zerstörungen verursachen, die die schlimmsten Hochwässer übertreffen.

\section{Stauseen und Landschaftsbild}

In jedem Klimabereich, besonders aber in Trockenländern, wo Vegetation und Wasserflächen zu den Seltenheiten gehören, sind Seen eine Bereicherung des Landschaftsbildes. Für Stauseen mit ihrer wechsel- und reizvollen Ufergestaltung gilt das in sehr verstärktem Maße, freilich nur unter der Voraussetzung, daß keine allzu großen Spiegelschwankungen auftreten, die pflanzenleere, häßliche Uferstreifen trockenfallen lassen und am Seeanfang ein weites Talstück als braune, verschlammte Fläche bloßlegen. Von solchen Ausnahmen abgesehen, sind auch die ursprünglich sehr heftigen Bedenken des Natur- und Landschaftsschutzes zumeist gegenstandslos geworden und richten sich nur noch, und mit Recht, gegen die Sperren, vor allem die Mauern, von denen niemand behaupten kann, daß sie Zierden der Landschaft sind. Es mag aber ein Trost sein, daß diese Verunstaltung der Natur sich auf einen einzigen Punkt beschränkt, während die weiten Seen auf Riesenflächen einen unbestreitbaren und sich vielseitig günstig auswirkenden Gewinn bedeuten. Wenn etwa das Innere von Texas, das noch um die Jahrhundertwende so gut wie ohne Seen war, heute Hunderte von Stauseen aller Größen aufweist - aus welchem Grunde die Texaner mit Stolz von ihrem «Land der Seen, reden -, so ist das ein deutlicher Hinweis auf die Beliebtheit, deren sich die Stauseen im Volk erfreuen. Auf jeden Fall ist die Stauseewirtschaft eine landschaftgestaltende Erscheinung allerersten Ranges, deren Bedeutung von Jahr zu Jahr wächst. Man muß sie unbedingt als ein positives Element beurteilen.

\section{Örtliche Lage der Stauanlagen}

$Z_{w}$ ar sind für den Sperrenbau unter vielen anderen geomorphologische, geologische und hydrologische, dazu menschlich-wirtschaftliche Bedingungen maßgebend, die alle für den Geographen höchst aufschluß- und belangreich sind; aber es dürfte leider selten vorkommen, daß er bei der Planung maßgeblich mitwirkt. Für Talsperren am besten geeignet sind Talengen an einem Gefällsknick, wo oberhalb eine flache, lange Talweitung großen Stauraum verbürgt und unterhalb eine hohe Stufe die Kraftnutzung fördert. Für die Begründung der Sperre ist fester Fels, dessen Schotter- oder Moränenbedeckung ausgeräumt werden muß, Vorbedingung. Das gilt besonders auch für Flußsperren. Brauchbare Baustoffe, zumal für die Betonbereitung, sollten an Ort und Stelle vorkommen. Für den See ist undurchläßiger Untergrund sehr wichtig. Das ist in kristallinem Gestein am besten gewährleistet. Kalk ist wegen hoher Durchläßigkeit und 
Löslichkeit weniger günstig und erfordert unter Umständen Zementabdichtung. Ebenso unerwünscht ist Spaltenbildung durch bruchtektonische Beanspruchung, es sei denn, daß sie zwecks Hebung des Grundwasserspiegels der Umgebung förderlich wirkt. Moränenmaterial bietet stets eine gute natürliche Abdichtung. Selbstverständliche Voraussetzung sind langjährige hydrologische Messungen, die jede Überraschung ausschließen. Menschliche Bedingungen verschiedenster Art sind in reicher Fülle zu klären. Menschenleere Täler eignen sich am besten, während besiedelte Gebiete mit um so höheren Ablösungs- und Umsiedlungskosten belastet werden, je volkreicher sie sind. Für den Bau der Wolga-Stauseen mußte mehreren Hunderttausend Menschen eine neue Heimat geschaffen werden.

Die meisten hier nur kurz angedeuteten Bedingungen verlieren in dem Maße an Gewicht, je höher sich die Technik entwickelt und früher ungeahnte Leistungen ermöglicht.

\section{Zwecke der Stauanlagen}

Den $Z$ wecken im einzelnen nachzugehen und ihre Auswirkungen in allen Verzweigungen zu verfolgen, ist eine echt geographische, sehr lohnende und noch viel zuwenig verfolgte Aufgabe. Sie sind zahlreich und räumlich äußerst verschieden. Hier steht der eine, dort der andere je nach den menschlichen Bedürfnissen und den klimatischen und hydrologischen Bedingungen im Vordergrund. Oft findet man Einzweckanlagen, weit überwiegend aber und namentlich bei großen Werken Mehr-, ja Vielzweckanlagen. Wenn man von Nebensächlichem absieht - wie etwa der Verbesserung der hygienischen Verhältnisse durch Überstauung von Malariaherden oder der Förderung der Selbstreinigung von Flüssen durch gesteigerte Frischwasserzufuhr -, schälen sich bei weltweiter Schau sechs nach ihrer Bedeutung geordnete Zwecke der Stauseen heraus:

1. Bewässerung steht weit an der Spitze. Sie ist eine schon in vorgeschichtlicher Zeit geübte Kunst, die die ältesten Stauanlagen geschaffen hat. Ihretwegen entstanden in den trockenwarmen Ländern, wo sie die Hautprolle spielt, unzählige Stauseen; ebenso zahllose treten heute und in der Zukunft hinzu, weil die Ernährung der Menschen durch Bewässerung am besten gesichert wird. Wie die USA unter allen Ländern der Erde mit der Zahl ihrer Stauseen weit an der Spitze stehen, so haben sie in der trockenen Westhälfte ihres Landes und zunehmend auch weiter im Osten eine Unzahl, die der Bewässerung und vielfaoh einzig und allein dieser dienen. Im übrigen sind sie in Indien, Australien, Ägypten, im Mittelmeergebiet, in China, in der Sowjetunion und vielen anderen Ländern von größter Bedeutung. Die reißende Zunahme der Bewässerung überall auf der Erde ist in erster Linie dem Bau von Stauanlagen zu danken. Wenn durch den einzigen Stausee Zimljansk am Don (1952, 21850 Mio. m³, $\left.2700 \mathrm{~km}^{2}\right) 27500 \mathrm{~km}^{2}$ in Bewässerungsland verwandelt werden können, so ist das doch ein sehr beachtlicher Erfolg.

2. Wasserkraftgewinnung hat den zweiten Rang. Sie war der ursprüngliche Anlaß der neuzeitlichen Entwicklung des Sperrenbaus. Ihre Bedeutung kann nicht überschätzt werden. Ist sie doch dadurch unentbehrlich, daß nur der Absatz elektrischen Stromes, der an jeder und an der kleinsten Sperre gewonnen werden kann, die meisten Stauanlagen erst wirtschaftlich tragbar, ja gewinnbringend macht. Es gibt sehr wenige Werke, bei denen das abfließende Wasser nicht auch die Turbinen eines Kraftwerkes treibt. In wasserreichen Ländern aber, wie im alpinen Frankreich, in der Schweiz, Italien, Österreich, Schweden, Norwegen, die alle unter Brennstoffmangel leiden, ist die Kraftgewinnung Alleinzweck. Ob sie freilich dem Wettbewerb der in Aussicht stehenden Entwicklung der Atomkraft standhalten kann und ob dadurch viele Stauanlagen zweckentfremdet werden, muß die Zukunft lehren.

3. Hochwasserschutz und im Zusammenhang damit Bodenkonservierung, also Schutz vor Abtragung und Wiedergutmachung der durch die Waldverwüstung verschuldeten 
Schäden, kommen nächstdem in Frage. Die Stauseen fangen Hochwasserwellen auf, lassen sie allmählich ablaufen und bannen so die Gefahr um so besser, je flächengrößer sie sind. Wenn es in den USA gelang, bei der Hochflut Mitte Januar 1950 allein durch die am Ohio und am Tennessee erbauten Stauanlagen bei Cairo (Illinois) eine Senkung des Mississippi-Spiegels um $77 \mathrm{~cm}$ zu erreichen und die Überflutung der Deiche zu verhindern, so ist das ein bemerkenswerter Erfolg, um so mehr, als sich dieser Betrag 1958 auf $100 \mathrm{~cm}$ erhöhte. Das regte dazu an, auch andere Stromgebiete in gleicher Weise zu schützen.

4. Versorgung mit Trink-und Brauchwasser ist ein immer dringlicheres Erfordernis, je mehr die Menschenzahl wächst und sich in Städten eng zusammendrängt. Der allzu rasch steigende Bedarf kann vielfach aus natürlichen Quellen nicht mehr gedeckt werden, nur Stauseen schaffen Abhilfe. Das spielt in Trockenländern eine besonders wichtige Rolle. Im Ruhrgebiet und im Raume von New York oder Los Angeles gibt es zahlreiche Anlagen, die nur diesem $Z$ wecke dienen.

5. Schiffahrtförderung auf den zu zusammenhängenden Staustrecken ausgebauten Strömen ist ein weiteres zunehmend wichtiges Ziel. Eine Kette von Stauseen macht aus einem schlecht schiffbaren Fluß eine erstklassige Großschiffahrtstraße. Oberhalb einer Sperre entsteht oft auf einigen hundert Kilometern ein hindernisfreier Fahrweg. Unterhalb kann schon eine Erhöhung des Niedrigwassers um 10 oder $20 \mathrm{~cm}$ förderlich sein. Auf dem Tennessee in den USA hob sich der Verkehr durch solche Maßnahmen von 1933 bis 1958 auf das 26 fache. Diese Erfolge ermutigen dazu, immer neue Flüsse durch Sperren der Großschiffahrt zu erschließen.

6. Erholungszwecke (Recreation) spielen eine ungeahnt wichtige und rasch weiter wachsende Rolle, sobald durch Straßenbauten und andere Anlagen günstige Voraussetzungen geschaffen sind. Besonders geeignet sind Seen, deren Spiegel wenig schwankt und die hohe landschaftliche Reize vermitteln. In Betracht kommen Ausflugsverkehr, Sommerfrischen, Badebetrieb, Angel-, Ruder-, Segel- und Motorsport mit vielen Begleiterscheinungen wie Gasthäusern, Häfen, Werften, Grundbesitzerwerb, Hausbau und so weiter. Bei manchen USA-Stauseen begegnet man außer 〈Recreation〉 dem Zweck Fish and Wildlife, was auf ihre besondere Bedeutung für Fischfang und Jagd hinweist. In den USA spielt der $W$ ater Playground bei vielen Stauseen eine ausschlaggebende, ja nicht selten alleinige Rolle, die noch vor 30 Jahren unmöglich vorauszusehen war. Eine heute sicherlich bereits weit überholte Erhebung an 287 bundesstaatlichen Seen zeigt folgendes Bild:

$\begin{array}{lccc} & \text { Jahr } & \text { Zahl der Seen } & \text { Besuchertage } \\ \text { Corps of Enginecrs } & 1946 & 50 & 5000000 \\ & 1951 & 101 & 21275000 \\ & 1960 & 157 & 109000000 \\ \text { Tennessee Valley Authority } & 1946 & 12 & 7000000 \\ & 1951 & 21 & 18400000 \\ & 1960 & 29 & 42300000 \\ \text { Bureau of Reclamation } & 1946 & ? & ? \\ & 1951 & 69 & 6500000 \\ & 1960 & 101 & 24300000\end{array}$

\section{Erdweite Verteilung der Stauanlagen}

Die sehr ungleichmäßige Verteilung sei durch zwei Tabellen veranschaulicht, deren eine die zeitliche, mit der Annäherung an unsere Zeit immer stürmischer wachsende Entwicklung, die andere die räumliche, nach Ländern gegliederte Anordnung zeigt. 


\begin{tabular}{|c|c|c|c|c|c|}
\hline Vor 1800 & 450 & & & & \\
\hline $1800-1849$ & 117 & & & & \\
\hline $1850-1899$ & 527 & & & & \\
\hline $1900-1949$ & 3583 & Davon & $1900-1919$ & & 917 \\
\hline & & & $1920-1939$ & & 1873 \\
\hline & & & $1940-1949$ & & 793 \\
\hline 1950-Ende 1962 & 2778 & Davon & $1950-1959$ & & 2009 \\
\hline & & & $1960-$ End & & 769 \\
\hline Summe & 7455 & davon & Erddämme & 4224 & \\
\hline Ende 1962 im Bau & 811 & davon & Erddämme & 450 & \\
\hline Ende 1962 geplant & 1049 & davon & Erddämme & 573 & \\
\hline Summe & 9315 & davon & Erddämme & 5247 & \\
\hline
\end{tabular}

Verteilung der Stauseen auf Länder (nach World Register)

\begin{tabular}{|c|c|c|c|c|c|c|}
\hline Land (Beginn des Sperrenbaus) & $\begin{array}{c}\text { Bestand } \\
\text { Ende } \\
1962\end{array}$ & $\begin{array}{c}\text { Davon } \\
\text { über } \\
1 \mathrm{~km}^{3}\end{array}$ & $\begin{array}{c}\text { Im Bau } \\
\text { Ende } \\
1962\end{array}$ & $\begin{array}{c}\text { Davon } \\
\text { über } \\
1 \mathrm{~km}^{3}\end{array}$ & $\begin{array}{c}\text { Geplant } \\
\text { Ende } \\
1962\end{array}$ & $\begin{array}{c}\text { Davor } \\
\text { über } \\
1 \mathrm{~km}^{\mathrm{g}}\end{array}$ \\
\hline Belgien (1876) & 5 & - & - & 一 & - & 一 \\
\hline Dänemark (1952) & 3 & - & 1 & - & - & - \\
\hline Deutsche Bundesrepublik (1721) & 64 & - & 2 & - & 6 & 一 \\
\hline Finnland (1929) & 28 & 2 & 2 & 1 & - & 一 \\
\hline Frankreich (1675) & 263 & - & 16 & - & 22 & 1 \\
\hline Großbritannien (1794) & 408 & - & 19 & - & 8 & 一 \\
\hline Island (1945) & 3 & - & - & - & - & 一 \\
\hline Italien $(1830)$ & 342 & - & 26 & - & 7 & 一 \\
\hline Jugoslawien (1898) & 40 & - & 9 & - & - & 一 \\
\hline Luxemburg (1958) & 3 & - & - & 一 & 一 & 一 \\
\hline Norwegen $(1890)$ & 87 & $?$ & 19 & ? & - & 一 \\
\hline Österreich (1911) & 40 & - & 4 & - & - & 一 \\
\hline Polen (19o7) & 11 & - & 2 & - & 3 & - \\
\hline Portugal (1920) & 41 & 1 & 5 & - & - & 一 \\
\hline Schweden (1908) & 81 & 1 & 12 & 2 & 11 & \\
\hline Schweiz (1895) & 73 & - & 21 & - & - & 一 \\
\hline Spanien (seit römischer Zeit) & 312 & 3 & 57 & 2 & 51 & 3 \\
\hline Sowjetunion (1926) & 44 & 21 & 13 & 8 & 8 & 2 \\
\hline USA (1836) & 2632 & $\begin{array}{c}81 \\
(1951: 62)\end{array}$ & 217 & 29 & 583 & 56 \\
\hline Argentinien (1923) & 29 & - & - & - & - & - \\
\hline Brasilien (19o1) & 176 & 9 & 27 & 5 & 31 & 9 \\
\hline Kanada (1832) & 293 & 23 & 12 & 4 & 36 & 5 \\
\hline Kolumbien (1938) & 12 & 一 & 2 & - & 1 & 一 \\
\hline Mexiko (1909) & 88 & 9 & 12 & 4 & - & 一 \\
\hline Uruguay (1946) & 3 & 1 & - & - & - & 一 \\
\hline Ceylon (187o) & 39 & - & - & - & - & 一 \\
\hline Indien (10. Jahrhundert) & 334 & 5 & 178 & 15 & 113 & 13 \\
\hline Japan (2. Jahrhundert) & 1705 & - & 122 & - & 146 & 一 \\
\hline Libanon (1954) & 4 & 一 & - & - & - & 一 \\
\hline Malaysia (1898) & 8 & - & 2 & - & - & 一 \\
\hline Pakistan (1913) & 8 & 1 & - & - & - & 一 \\
\hline Philippinen (1929) & 5 & - & - & 一 & 2 & 一 \\
\hline Syrien (1960) & 2 & - & 一 & - & - & 一 \\
\hline Thailand (?) & - & - & 1 & 1 & 3 & 3 \\
\hline Türkei (1723) & 23 & 4 & 5 & - & 4 & - \\
\hline Vietnam (1938) & 3 & - & 1 & - & 3 & \\
\hline
\end{tabular}




\begin{tabular}{|c|c|c|c|c|c|c|}
\hline Land (Beginn des Sperrenbaus) & $\begin{array}{c}\text { Bestand } \\
\text { Ende } \\
1962\end{array}$ & $\begin{array}{c}\text { Davon } \\
\text { über } \\
1 \mathrm{~km}^{3}\end{array}$ & $\begin{array}{c}\text { Im Bau } \\
\text { Ende } \\
1962\end{array}$ & $\begin{array}{c}\text { Davon } \\
\text { über } \\
1 \mathrm{~km}^{3}\end{array}$ & $\begin{array}{c}\text { Geplant } \\
\text { Ende } \\
1962\end{array}$ & $\begin{array}{c}\text { Davon } \\
\text { über } \\
1 \mathrm{~km}^{3}\end{array}$ \\
\hline Ägypten (19o2) & 2 & 2 & 1 & 1 & - & - \\
\hline Rhodesien (19o1) & 26 & 2 & 2 & - & 1 & - \\
\hline Sudan (1925) & 1 & - & 2 & 2 & - & - \\
\hline Tunesien (1925) & 5 & - & 4 & - & 12 & - \\
\hline Australien (1861) & 203 & 7 & 18 & 2 & 15 & 3 \\
\hline Neuseeland (1872) & 38 & 1 & 3 & 1 & - & - \\
\hline
\end{tabular}

\section{Kurze Kennzeichnung der Erdteile}

Die vorstehende Zusammenstellung läßt trotz vielen Lücken und Unzulänglichkeiten wichtige Tatsachen erkennen. Überall schreitet der Bau von Stauanlagen rasch fort. Er wird auch in Zukunft nicht nachlassen, da die unheimlich wachsende Menschenzahl dazu zwingt, mit dem Wasser haushälterisch umzugehen und es vor allem für

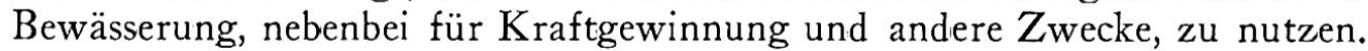

Europa ist die Heimat der neuzeitlichen Stauseewirtschaft. Sie ist hier hochentwickelt, aber immer noch sehr ausbaufähig. Kennzeichnend sind viele kleine und mittelgroße Seen, während Riesen fehlen. Das ist bedingt durch die Raumnot des dichtbevölkerten Erdteils. Es ist typisch, daß die größten Seen in weiträumigen, schwach besiedelten Ländern liegen (Schweden, Spanien). Bewässerung ist das Hauptanliegen der südlichen Halbinseln. In den übrigen Räumen steht Kraftgewinnung weit im Vordergrund. In Deutschland kommt die Förderung der Schiffahrt sowie die Befriedigung des steigenden Wasserbedarfs im Rhein-Ruhr-Gebiet maßgeblich hinzu.

In der Sowjetunion ist Raumnot unbekannt. So besitzt sie zwar wenige, aber gigantische Stauseen. Sie dienen in erster Linie der Kraftgewinnung, dann der Schiffahrtförderung, im Süden der Bewässerung von Steppen und Halbwüsten. Dazu ist die Aufhöhung des bedrohlich sinkenden Kaspi-Spiegels ein wichtiges Ziel. Die russischen Sperren sind fast nur Erddämme, denen eine das Kraftwerk tragende Mauer eingefügt ist. Ende 1962 verfügte die Sowjetunion über einen Totalstauraum an fertigen und im Bau befindlichen Seen von $535+123 \mathrm{~km}^{3}$, während $31 \mathrm{~km}^{3}$ geplant waren. Die Summe $689 \mathrm{~km}^{3}$ spricht deutlich für die Riesenhaftigkeit der an Zahl geringen Stauseen. Das Programm sieht viele weitere Großbauten vor.

Die Vereinigten Staaten sind das höchstentwickelte und als Vorbild unerreichte, mit Riesenschritten weiter fortschreitende Stauseeland der Erde. Es gibt Anlagen jeder Größe, weil unbesiedeltes Land reichlich zur Verfügung steht. Was in Europa groß ist, ist hier klein. Kennzeichnend ist die Vielzahl der Zwecke. Mit der Formel «Bewässerung im Westen, Kraftgewinnung im Osten> ist es nicht mehr getan. Hochwasserschutz wird mit höchster Energie betrieben und schreitet rasch fort. Er ist die allererste Voraussetzung der dringend notwendigen Bodenkonservierung. Schiffahrtförderung ist nicht weniger wichtig, der eine unvorstellbare Belebung des Flußverkehrs zu danken ist. Wasserversorgung der Ballungsräume der Bevölkerung und Erholungszwecke sind weitere wichtige Ziele. Anfang 1947 rechnete man mit einem gesamten nutzbaren Stauraum von $201 \mathrm{~km}^{3}$. Anfang 1957 stieg der Totalstauraum der fertigen und im Bau befindlichen Sperren auf $499 \mathrm{~km}^{3}$. Für die geplanten Seen, die heute zumeist fertiggestellt sind, ergaben sich $237 \mathrm{~km}^{3}$. Das sind im ganzen $736 \mathrm{~km}^{3}$. Da der mittlere Jahresniederschlag des USA-Kerngebietes auf rund $5600 \mathrm{~km}^{3}$ geschätzt wird, ergibt sich, daß davon rund 13 Prozent von Stauseen aufgenommen werden können. Ihre Gesamtfläche ist heute mit $60000 \mathrm{~km}^{2}$ eher zu niedrig als zu hoch anzugeben.

In den übrigen Ländern der Neuen Welt bemüht man sich ebenfalls kräftig um die Ausweitung der Stauseewirtschaft. Großanlagen sind zahlreich. Bewässerung ist Trumpf. Eine Ausnahme bildet Kanada, wo Kraftgewinnung weit an der Spitze steht. Ein Sonderfall ist der Gatunsee, der dem Verkehr durch den Panamakanal dient. 
Die Stauseen Asiens, Afrikas und Australiens stehen noch im Anfang einer unabsehbaren Entwicklung. Sie dienen sämtlich der Bewässerung, wenn auch Kraftgewinnung nebenhergeht und in China die Bändigung des Hwangho ein vordringliches Ziel ist. Japan besitzt zahllose Kleinanlagen und überschritt erst 1904 die Grenze von 1 Mio. $\mathrm{m}^{3}$; erst in den letzten Jahrzehnten entstanden auch größere Werke. Indiens Stauseewirtschaft wird zielbewußt in großem Maßstab rasch weiter ausgebaut, weil Bewässerung dort eine Lebensfrage ist. Großanlagen stehen im Vordergrund des Interesses. Ende 1962 rechnete man bei fertigen, im Bau und in der Planung begriffenen Sperren mit einem Gesamtstauraum von $173 \mathrm{~km}^{3}$. Afrika ist der Erdteil allergrößter Möglichkeiten. Das Beispiel des Akosombo am Volta und des Elizabeth Lake am Sambesi wird viele Nachfolger von ähnlichem Rang finden. Australiens Stauseen liegen auf der Ostseite des Kontinents und sind zum Teil dadurch bemerkenswert, daß sie ihr Wasser aus Flüssen der regenreichen Außenflanke des Gebirges zapfen und durch lange Stollen der trockenen Innenseite zu führen.

\title{
Schlußbetrachtung
}

Meine kurzgefaßten Darlegungen sollten zeigen, welch vielseitige Aspekte den Geographen mit den Stauanlagen verbinden. Sie sollten dazu anregen, daß sich die Fachgenossen mehr als bisher dieser Fragen annehmen. Wenn man von dankenswerten Einzelbemühungen absieht, ist in der Vergangenheit allzu wenig in dieser Richtung geschehen. Es ist wichtig, das äußerst beziehungsreiche Wesen der Stauseen zu erforschen und die spärlichen, zurückhaltenden Nachrichten der Ingenieure zu ergänzen und lebendig $z u$ machen.

\section{THE WORLD'S BARRAGES}

A discussion on the world's barrages must of necessity be based on engineering data: it is perhaps unfortunate that even this appears incomplete for a relevant geographic analysis.

The author indicates the importance and relevance to geography as such: these edifices, and the artificial lakes that go with them must surely be the greatest transformation of the natural landscape that man has accomplished. This quite apart from the geographical significance of their multifold applications: irrigation, hydro-electric power, flood control etc. 'The essay is concluded by an inspection of the important factors that determine the global distribution of some 10000 barrages.

\section{LA SECTION DE GÉOGRAPHIE ET CARTOGRAPHIE A LA I45e SESSION ANNUELLE DE LA SHSN}

\author{
ERNES'T PAILLARD
}

La Société helvétique des sciences naturelles a célébré à Genève, les 24, 25 et 26 septembre, le $150 \mathrm{e}$ anniversaire de sa fondation. Parmi les quelque cinq cents participants, et 18 sections, qui affluèrent aux manifestations, les géographes de la Fédération furent discrets, tout en faisant preuve d'une grande activité.

Samedi, dès 9 heures, se déroula le programme des conférences. Local de la séance: Université, salle 49. Présidence: E. L. Paillard, Lausanne. Toutes les sections étaient représentées, à l'exception de Neuchâtel.

Le nombre élevé des communications (voir la liste ci-dessous) engagea les délégués à ne pas tenir d'assemblée générale en fin de matinée, mais à la reporter au mois de décembre, à l'occasion de la transmission des pouvoirs du Comité central. 